\title{
Analyse d'un incident radiologique et des leçons à en tirer : le cas d'un incident en radiographie industrielle en Grande-Bretagne
}

\author{
J. CROFT*; traduit par Ch. LEFAURE**
}

(Manuscrit reçu le 25 mai 2000, accepté le 25 mai 2000)

RÉSUMÉ Cette note présente une étude de cas qui analyse les leçons à tirer en matière de radioprotection à partir d'un incident radiologique en vue de prévenir l'apparition d'incidents du même type. Cette note a été publiée en anglais dans la European ALARA Newsletter $n^{\circ} 2$ (janvier 1997) et elle synthétise un des exemples recensés dans la base de données anglaise IRID (Ionising Radiation Incident Database) du «Health and Safety Executive», «National Radiological Protection Board» et de l'«Environmental Agency».

\begin{abstract}
This note describes a case study to provide feedback analysis and lessons to be learned from a radiological incident. This one occurred in the UK. It has been published in the European ALARA Newsletter no. 2 (January 1997) and has been selected from the IRID database (Ionising Radiation Incident Database) managed by the Health and Safety Executive, National Radiological Protection Board and Environmental Agency.
\end{abstract}

\section{L'incident}

Un appareil automatisé de radiographie industrielle, contenant une source d'Iridium 192 de $550 \mathrm{GBq}$, a été utilisé pour examiner une soudure circonférentielle sur un poste de travail «ouvert», c'est-à-dire dans une zone non strictement close dans une usine. Après examen de la soudure, le mécanisme de rappel a été utilisé pour ré-introduire la source dans son conteneur. Pendant le déroulement de cette procédure, le débit-mètre portable, situé près du mécanisme de rappel a enregistré une forte baisse du débit de dose, les travailleurs ont alors fait l'hypothèse que cela signifiait que la source était revenue dans le conteneur. Cependant, cinq jours plus tard, l'appareil a été réutilisé et tous les clichés étaient blancs, ce qui a révélé

\footnotetext{
* NRPB, Chilton, Didcot, Oxon OX11 ORQ, UK.

** CEPN, BP 48, 92263 Fontenay-aux-Roses Cedex, France
} 
que la source avait disparu de son container; à l'évidence la source était perdue. Après recherche, elle a été retrouvée près du lieu de sa dernière utilisation. Des investigations ultérieures ont montré que la source s'était détachée de son câble conducteur, pour une raison inconnue, et qu'elle était tombée, sans que quiconque s'en aperçoive, du tube guide lors du démontage de l'appareil.

Le débit-mètre n'a pas été utilisé correctement pour vérifier que la source était retournée dans le conteneur. La chute de débit de dose observée auprès du mécanisme de rappel n'était intervenue que parce que la source s'était détachée du câble conducteur à proximité du conteneur qui a joué un rôle d'écran entre la source et le débit-mètre. La source a été retrouvée d'une façon contrôlée par la personne compétente. On s'est rendu compte que pendant ces cinq jours, 78 personnes ont été exposées à des degrés divers. Les estimations concernant leurs doses sont fournies ci-dessous. La source était restée dans un emplacement facilement accessible et si elle avait été ramassée pendant ces cinq jours cela aurait entraîné des effets déterministes, éventuellement létaux.

\begin{tabular}{|c|c|}
\hline Nombre de travailleurs exposés & Dose (dose efficace mSv) \\
\hline 2 & $100-150$ \\
4 & $30-100$ \\
9 & $11-30$ \\
63 & $<11$ \\
\hline
\end{tabular}

\section{Leçons}

1- Le débit-mètre aurait dû être ramené près du conteneur pour vérifier que la source était bien revenue en position de sécurité dans celui-ci.

2 - Des mesures devraient systématiquement être effectuées avant l'entreposage du matériel en stockage. Cela permettrait de déterminer beaucoup plus tôt que la source est manquante.

\section{RÉFÉRENCE}

Croft J.R., Thomas G.O., Walker S., Williams C.R. (1999) IRID, Ionising Radiations Incident Database ; first review of cases reported and operation of the database. NRPB - 135 pages. 